\title{
Drug Trials in Children: Ethical, Legal, and Practical Issues
}

\author{
Ralph E. Kauffman, MD
}

\begin{abstract}
nvolvement of children in clinical trials of drugs entails issues and considerations that are unique to children. This discussion focuses on selected ethical issues of particular importance when engaging in clinical trials in children, briefly reviews some legal considerations, and concludes by discussing certain practical issues which impact on pediatric clinical trials.
\end{abstract}

\section{ETHICAL ISSUES}

\section{History}

General societal concern regarding the ethics of human experimentation is a recent phenomenon. Before World War II, clinical trials in general, and clinical trials in children, were conducted in a rather informal manner with little public scrutiny. Edward Jenner first tested his smallpox vaccines on his firstborn son and on neighbors' children. Caroline of England arranged for Jenner's vaccine to be tested in children from a foundling hospital before it was administered to the royal children. When Joseph Meister contracted rabies and was brought to Louis Pasteur, Pasteur consulted with two medical colleagues and then intervened at the point that everyone agreed "the death of the child appeared inevitable."

The Nuremberg trial of Nazi doctors resulted in a code of ethics for human experimentation. However, the Nuremberg code ${ }^{1}$ did not specifically address research in children and received little attention before the late 1960s.

In 1964, the need for special protection and surrogate decision making for subjects with limited capacity to consent was recognized in the World Medical Association's Declaration of Helsinki. ${ }^{2}$

From the Division of Clinical Pharmacology/Toxicology, Wayne State University, Children's Hospital of Michigan, Detroit, Michigan. Address for reprints: Ralph E. Kauffman, MD, Division of Clinical Pharmacology/Toxicology, Wayne State University, Children's Hospital of Michigan, 3901 Beaubien Boulevard, Detroit, MI 48201.
Dr. Henry Beecher published a paper in the New England Journal of Medicine in $1966^{3}$ on Ethics and Clinical Research in which he censured 22 human studies published in prestigious U.S. journals for violating basic ethical principles of human research. One of the objects of Beecher's criticism was a study of viral hepatitis, published in the NEJM (1958), ${ }^{4}$ performed at Willowbrook State School in which 51 mentally retarded children were purposefully fed infectious fecal extracts to study viral hepatitis. Beecher's paper was followed by an outcry from multiple facets of society alleging unethical practices and violations of individual human rights during the conduct of medical research. This culminated in 1974 in the establishment of the National Commission for the Protection of Human Subjects of Biomedical and Behavioral Research. The report of the National Commission was published in 1978. ${ }^{5}$

Concurrently with the National Commission's work, the Committee On Drugs of the American Academy of Pediatrics, under contract with the FDA, developed guidelines for the ethical study of drugs in infants and children which were published in $1977 .^{\circ}$ The COD guidelines recently have been revised and will be published in late 1993 or early 1994. Both the HHS and AAP documents have had a profound impact on conduct of drug studies in children during the past decade.

\section{Ethical Considerations When Doing Studies in Children}

The regulations and guidelines for the protection of all human subjects arise from three basic premises:

1. Respect for the rights of the individual;

2. The obligation to protect the individual from undue risk; and

3. Fairness in distribution of the burdens and benefits of research.

Children comprise a particularly vulnerable population that must be afforded protections over and above those provided to autonomous adults because of their limited cognitive abilities compared with 
normal adults and their dependency on responsible adults. Several areas are of particular importance to children.

Informed Consent. The principle of respect for the rights of the individual demands that the individual not be a research subject without being informed about the benefits and risks of the research and freely giving permission to participate as a research subject. However, children are not recognized under law as autonomous individuals, although they do enjoy individual rights. In addition, they do not possess the intellectual maturity to independently consent to participate as research subjects. Therefore, consent must be given by a surrogate, e.g. a responsible adult who usually is the parent. In doing so, it is assumed that the surrogate is acting in the best interests of the child and is not influenced by factors that may not be in the child's interest. Investigators need to be sensitive to situations in which the legal surrogate consentor may not be acting solely in the best interests of the child.

To the extent they are capable, children should be allowed to participate in the consent decision along with the surrogate consentor. Consent of children $>13$ years should be obtained unless there is an overriding reason why this should not be done. Also, children $>7$ years of age should have the right to say "no" to participation, unless doing so would clearly not be in their best interest. The Academy of Pediatrics Statement has referred to this as "assent."

Risk/Benefit. Considerations of risk assume that the study is well designed and has scientific merit. Participation in poorly designed or scientifically nonmeritorious studies can never be defended as ethical regardless of how little risk is involved.

In most instances the risk to child subjects is reduced by commencing studies in children after animals and initial studies in adults have documented acceptable safety. In some cases this is not possible; for example when the treated condition only occurs in children and/or the drug is too toxic to study in adult volunteers.

Because of their particular vulnerability and inability to independently consent to participate as research subjects, children are provided extra protection against undue risks. Both the Federal and Academy of Pediatrics guidelines have defined risk in terms of minimal risk and greater than minimal risk. Risk is relative and must always be defined in relation to possible benefits from participation. Some risk factors that deserve special attention for children are fear of a procedure, pain, and separation from parents or familiar surroundings.

Children should be permitted to participate in studies involving minimal risk if the study promises benefit to the larger society of children, to a specific group of children, and/or to the individual child.

With rare exception, participation in research involving greater than minimal risk should be restricted to studies presenting the prospect of direct benefit to the individual subject. In this case, the relationship of the anticipated benefit to the risk should be at least as favorable as that presented by available alternative therapies. Typically such studies are carried out in a therapeutic context, e.g., the child is participating in a study as a part of their medical care.

Selection of Subjects. No particular socioeconomic, racial, or ethnic group within larger society should participate disproportionately in a clinical trial unless the selection is a necessary part of the study (i.e., study of cystic fibrosis, European Americans or sickle-cell anemia, African Americans). Likewise, no particular group should be excluded from the benefits of a clinical trial unless the exclusion is dictated by the inherent characteristics of the population and/or the purpose of the study.

A related issue is compensation for participation. While reimbursement for direct expenses, time, and inconvenience associated with participation may be appropriate, compensation which results in unfair inducement to participate by a particular segment of the population should be avoided. In addition, it is important to avoid incentives sufficient to induce surrogates to consent for a dependent to participate in a study or subject them to painful or invasive procedures.

Special Populations. Certain populations of children deserve special mention because they are more vulnerable than the general population of children and therefore must be afforded special protection.

Institutionalized children, both handicapped and non-handicapped, are at increased risk for exploitation. Furthermore, surrogate consent presents special problems in this population. Therefore, these children should not be included in clinical trials unless they directly benefit from participation and/or the subject of the trial pertains to their special circumstance of being institutionalized. On the other hand, such children should not be excluded from therapeutic trials from which they could directly benefit. Additional protection may be provided by an advocate who is independent of the investigator, institution, and legal guardian.

Emancipated minors comprise another special population. In many states emancipated minors are legally qualified to independently give consent for medical treatment for themselves. It follows that 
they may be qualified to give independent consent to participate in studies pertaining to the medical condition for which they can legally give consent for treatment. This is a delicate issue and the investigator is advised to rely heavily on critical review and supervision by the responsiblo IRB if this is done.

Children with permanently debilitating or lethal diseases are uniquely dependent and vulnerable. Children with malignancies and children with HIV infection illustrate this dilemma. In some cases potentially efficacious drugs can only be studied in this population. Many of the investigational protocols result in substantial morbidity and suffering. Can one always assume the surrogate consentor is making the best decision for the child? At what point does the child have a right to refuse to participate?

HIV infection poses additional problems because of the stigmata attached to it by society. How does one maintain anonymity for the child and parents? Because HIV infection is predominantly associated with high-risk lifestyles, how does this affect the ability of the parent to act as a responsible surrogate for the child?

Investigation involving these special groups requires particular diligence on the part of the investigator and IRB to be sure the child is adequately protected and at the same time has access to the benefits of available research studies.

\section{LEGAL ISSUES}

Legal considerations impinge on drug trials in children at several levels. These include pertinent Federal regulations, labeling requirements, and civil liability.

\section{Federal Regulations}

Investigators and sponsors of clinical trials must, of course, be in compliance with applicable federal statutes and regulations that govern research in human subjects. Among other things, HHS regulations require that studies to be conducted in human subjects be reviewed and approved by an Institutional Review Board. This is one of the ways society has formalized its obligation to protect the rights and welfare of the human subject. The regulations also require that informed consent be obtained before participation. In the case of the dependent child, surrogate consent must be obtained, usually from the parent or legal guardian. Criminal law may also speak to the issue of consent. In many jurisdictions, the physician may be committing battery if any treatment or procedure is administered without consent.

The FDA has never implemented regulations specifically governing testing of drugs in children, al- though guidelines do exist. They have relied on the HHS regulations and reviewed pediatric issues on a case by case basis.

\section{Civil Liability}

There seems to be widespread concern on the part of industry sponsors that clinical trials in children are associated with substantially greater liability risk than trials involving adults. However, litigation as a direct result of injury associated with clinical trials has been rare. Likewise, civil suits against clinical investigators have been rare. This appears to be somewhat uncharted territory with few legal precedents. However, to date, the fear of civil liability suits appears to be more real than the reality of actual suits. Although liability suits may not have significantly influenced the conduct of pediatric clinical trials, the concern about liability has been a deterrent to doing trials in children. Mr. Brian Yolles, an attorney speaking at the IOM workshop on Drug Development and the Pediatric Population, held April 23-24, 1990, pointed out that if HHS regulations or FDA or COD guidelines are not followed, there is potential for liability. IRBs also may share liability if guidelines are not followed. However, even if guidelines are followed there is still potential liability if courts and juries view the guidelines as the minimal standard.

On the brighter side, Mr. Yolles predicted that courts will be reluctant to impose liability on sponsors of well-controlled trials of drugs in children. He pointed out that testing of drugs has become recognized by society and by the courts as increasingly important. If drugs are to be used safely and effectively in children, they must be tested in children.

IRBs also are not immune to civil complaints on the basis of their approval or disapproval of study protocols. In 1973 the University of California was sued to revoke approval by their IRB of a 5-year allergy study which included normal children as controls. ${ }^{7}$ The suit was filed by a dissenting member of the IRB who alleged that normal children were being volunteered for invasive procedures as part of the study and payment to parents of $\$ 300$ constituted undue inducement to persons in economic distress.

\section{Labeling Requirements for Children}

Drugs cannot be labeled for use in children unless the statutory requirements for evidence of safety and efficacy are met. This requires that well-controlled trials be conducted in the age groups for which the drug is labeled. Because of lack of such trials and/or absence of such data in the New Drug Application, the majority of drugs on the market are not labeled 
for use in children. This places the physician caring for children in the untenable position of not prescribing many medications for children or prescribing off label. Frequently the latter is a necessary choice. When prescribing off label, it is important to recognize that FDA-approved labeling regulates the drug in interstate commerce, not medical practice. However, off label prescribing places an increased burden of proof on the physician to establish the scientific and medical basis for doing so. In this sense, it may increase the physician's liability potential. The physician may actually be in a stronger position with respect to liability when administering a medication off label under an approved protocol than when doing so as a routine prescription.

\section{PRACTICAL ISSUES}

The essential ingredients for successful clinical trials are: 1) investigator expertise; 2) clinical and technical facilities; 3) adequate patient population; and 4) funding.

Ethical considerations and technical constraints are frequently cited as impediments to pediatric studies. However, they contribute minimally to lack of pediatric research. An ethical framework within which to do pediatric studies has been in existence since 1977 and advances in technology have negated technical constraints as a rate-limiting factor. Although regulatory constraints have been a reality, the FDA has taken steps to address this issue. Therefore, ethical, technical, or regulatory issues are not major impediments to conducting clinical trials in children.

\section{Patient Population}

With rare exception, drug studies in children require access to a population of ill children for which the drug holds some promise of benefit. Availability of an appropriate patient population is most commonly the rate limiting step in successfully conducting studies in children. Optimal patient populations are most easily identified in large pediatric facilities. Some disease entities are rare enough that no center can enroll enough patients for a meaningful study within a reasonable time period. On the other hand, certain clinical trials may best be carried out in large ambulatory pediatric practices.

\section{Funding}

Clinical trials in infants and children tend to be more expensive than comparable trials in adults. Several factors contribute to this. For example, pediatric studies pose logistic challenges that require greater personnel time and effort than adult studies. Further- more, pediatric studies frequently are, of necessity, opportunistic. This tends to prolong enrollment time and increase cost.

Although pediatric studies may be more expensive, the incentive to fund pediatric studies is less. In the case of most pharmaceutical products, the pediatric share of the market is a small fraction of the total market. Because of this, along with liability concerns, there is limited economic incentive to invest a great deal in clinical studies in children. This has resulted in a majority of drugs entering the market place and finding their way into off label use in children with little or no data in children. The economic impediments to pediatric drug studies need to be reduced. Several recent initiatives promise to help address this issue. The FDA has proposed regulatory changes to facilitate inclusion of pediatric indications in labeling for new drugs, the NIH will be funding a pediatric clinical trials network, and proposed legislation is under review which would extend the period of exclusivity (patent protection) of new drugs for which clinical trials are done to support pediatric labeling.

\section{SUMMARY}

Technical limitations, logistic challenges, and ethical constraints no longer provide excuses for not conducting well-designed studies of therapeutic agents in children. In fact, to not do so is difficult to defend ethically. Clinical trials in children are feasible and essential for safe and effective use of medications in children. The time is long past when drugs with therapeutic potential in children should be marketed with labeling for use only in adults.

\section{REFERENCES}

1. United States Adjutant General's Department: Trials of War Criminals Before Nuremberg Military Tribunals Under Control Council Law No. 10 (October 1946 to April 1949). Washington, DC: U.S. Government Printing Office. 1947:2:181-183.

2. World Medical Association: Declaration of Helsinki (1964): The changing mores of biomedical research. Ann Intern Med 1965; 7:74-75 (supplement).

3. Beecher HK: Ethics and clinical research. NEJM 1966;274: 1354-1360.

4. Ward R, Krugman S, Giles IP, Jacobs AM, Bodansky O: Infectious hepatitis: Studies of its natural history and prevention. NEJM 1958;258:407-416.

5. The National Commission for the Protection of Human Subjects of Biomedical and Behavioral Research: The Belmont Report: Ethical principles and guidelines for the protection of human subjects of research. OPRR Reports 1979.

6. Committee on Drugs: Guidelines for the ethical conduct of studies to evaluate drugs in pediatric populations. Pediatrics 1977;60:91-101.

7. Pediatric Research Curb Sought in California - Children as Controls. Pediatric News 1973; 7:1,60. 